\title{
The relationship between decorrelation time and sample thickness in acute rat brain tissue slices (Conference Presentation)
}

\author{
Joshua Brake, Mooseok Jang, Changhuei Yang, California Institute of Technology (United \\ States)
}

\begin{abstract}
The optical opacity of biological tissue has long been a challenge in biomedical optics due to the strong scattering nature of tissue in the optical regime. While most conventional optical techniques attempt to gate out multiply scattered light and use only unscattered light, new approaches in the field of wavefront shaping exploit the time reversible symmetry of optical scattering in order to focus light inside or through scattering media. While these approaches have been demonstrated effectively on static samples, it has proven difficult to apply them to dynamic biological samples since even small changes in the relative positions of the scatterers within will cause the time symmetry that wavefront shaping relies upon to decorrelate. In this paper we investigate the decorrelation curves of acute rat brain slices for thicknesses in the range 1-3 mm (1/e decorrelation time on the order of seconds) using multi-speckle diffusing wave spectroscopy (MSDWS) and compare the results with theoretical predictions. The results of this study demonstrate that the $1 / \mathrm{L}^{\wedge} 2$ relationship between decorrelation time and thickness predicted by diffusing wave spectroscopy provides a good rule of thumb for estimating how the decorrelation of a sample will change with increasing thickness. Understanding this relationship will provide insight to guide the future development of biophotonic wavefront shaping tools by giving an estimate of how fast wavefront shaping systems need to operate to overcome the dynamic nature of biological samples.
\end{abstract}

View presentation recording on the SPIE Digital Library: http://dx.doi.org/10.1117/12.2208972.4848677972001 